\title{
Non-Cirrhotic Portal Hypertension in a Patient With Colonic Carcinoma Treated With Oxaliplatin
}

\author{
Maria Cynthia Fuentes-Lacouture ${ }^{\mathrm{a}, \mathrm{c}}$, Edgar Camilo Barrera-Garavito ${ }^{\mathrm{a}}$, \\ Andrea Gomez ${ }^{a}$, William Mantilla ${ }^{b}$
}

\begin{abstract}
Oxaliplatin is a chemotherapeutic agent with direct toxic action on deoxyribonucleic acid (DNA), which is known to cause an arrest in its synthesis and inducing cell death. It is a crucial medication for colorectal carcinoma, and in combination with other medications has demonstrated to exhibit synergism, managing to increase patients' survival, especially when compared to monotherapy with 5-fluoracil. Neurotoxicity is its most well-known adverse effect. However, other less frequent secondary effects have been described in case reports, among them liver injury, which is usually secondary to liver sinusoid injury. Despite the wide frequency of the use of this drug, the relationship of oxaliplatin with the development of portal non-cirrhotic hypertension is largely unknown, which translates into a sub-diagnosis, representing an additional risk to patients who develop this complication. We present the case of an adult patient, who during treatment with the FOLFOX scheme for colorectal carcinoma, presents signs suggestive of portal hypertension, without other risk factors besides the administration of oxaliplatin.
\end{abstract}

Keywords: Non-cirrhotic portal hypertension; Oxaliplatin; Pathophysiology

\section{Introduction}

Colorectal carcinoma is the fourth most common malignancy, and the second leading cause of death related to cancer in the USA [1]. In Colombia, colorectal carcinoma is the third most common malignancy (second among women) and the third cause of death by cancer, accounting for $9.7 \%$ of all cancer deaths in our country [2]. Medical treatment is based on

Manuscript submitted December 3, 2020, accepted December 10, 2020

Published online January 19, 2021

anternal Medicine Department, Fundacion Cardioinfantil, Bogota, Colombia ${ }^{\mathrm{b}}$ Hemato-Oncology, Fundacion Cardioinfantil, Bogota, Colombia

${ }^{\mathrm{c} C}$ Corresponding Author: Maria Cynthia Fuentes-Lacouture, Internal Medicine

Department, Fundacion Cardioinfantil, Bogota, Colombia.

Email: cynthiafuentes27@gmail.com

doi: https://doi.org/10.14740/jmc3630 chemotherapy, in which one of the main drugs is oxaliplatin, a deoxyribonucleic acid (DNA) disrupter, which is applied in early (as an adjuvant treatment after surgery) or advanced stages, and has managed to increase patients' survival, especially when compared with monotherapy with 5 -fluorouracil [2]. One of the best-known adverse effects of oxaliplatin is neurotoxicity (manifested as sensory neuropathy) [2]; however other less known effects have been reported, including sinusoidal liver injury, which manifests with changes related to portal hypertension.

Medications can trigger non-cirrhotic portal hypertension (NCPH) due to direct damage to liver or because of fibrosis induction. In both cases, sinusoidal and post-sinusoidal damage are the main histopathological findings. Among known chemotherapeutic medications, the most common are gemtuzumab, ozogamicin, dacarbazine, cytosine arabinoside, 6thioguanine, busulfan and cyclophosphamide [3]. However, there are few reports in literature about the relation between oxaliplatin administration and NCPH. Despite of the description about this association, pathophysiology is not completely elucidated, mainly due to the small number of cases reported. The under-diagnosis of this association is troublesome considering the high morbidity and mortality of NCPH.

We present the case of a female with past history of colorectal cancer, which was under treatment with oxaliplatin, and debuted with esophageal bleeding, in whom NCPH was diagnosed, and after extensive workout, it was finally attributed to oxaliplatin.

\section{Case Report}

A 55-year-old woman was admitted to the emergency department for dark stools on last day. In addition, she reported appearance of spontaneous hematomas on the left hand and right leg. Patient had a history of colon carcinoma pT3apN2bM1a IVA, metastatic to lung and liver, managed surgically, and was on fifth line of chemotherapy with FOLFOX + bevacizumab for 10 cycles. Her last chemotherapy cycle has been 20 days ago, with a history of hematologic toxicity secondary to multiple chemotherapy schedules. On physical examination, generalized mucocutaneous pallor was striking, and two hematomas were found (one of $20 \times 10 \mathrm{~cm}$ in the right quadriceps, another of $7 \times 7 \mathrm{~cm}$ in the left hand), both painful on palpation. Admission complete blood count revealed pancytopenia 


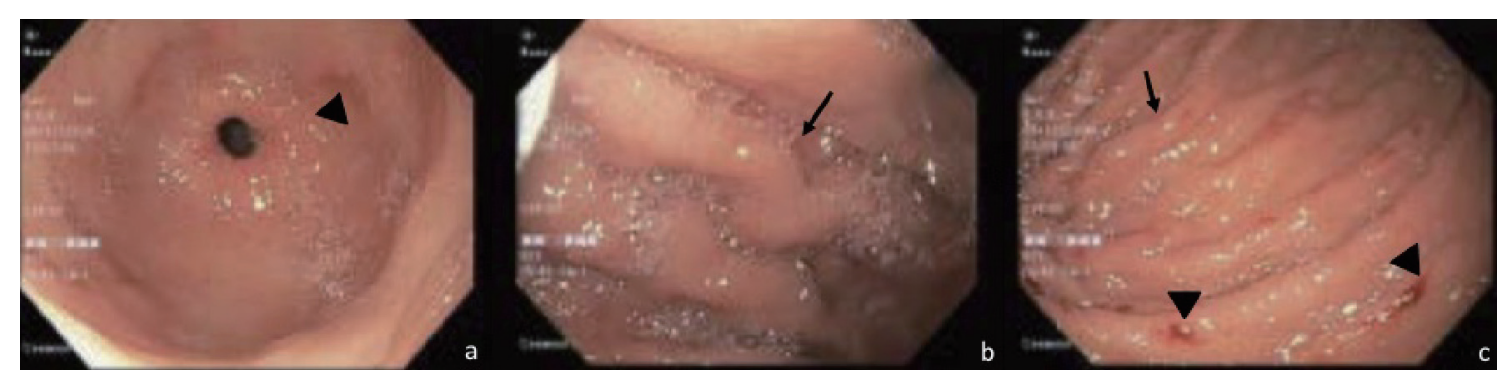

Figure 1. Large esophageal varices with red signs, changes compatible with hypertensive gastropathy, without active bleeding.

with total leukocytes of 3,250 cells $/ \mathrm{mm}^{3}$, hemoglobin $10.1 \mathrm{~g} /$ $\mathrm{dL}$, and 38,700 platelets. Kidney function was within normal limits. Because of severe thrombocytopenia and dark stools, a diagnosis of upper gastrointestinal bleeding was suspected (Blatchford score of 5 points (high risk), and a pre-endoscopic Rockall score of 2 points). Infusion of proton-pump inhibitor was started, and endoscopy of the upper digestive tract revealed changes compatible with portal hypertensive gastropathy and large esophageal varices with red dots and requirement for band ligation (Fig. 1 a-c). The patient had no known history of liver disease, and signs of chronic liver disease were absent in physical examination. Liver function tests revealed: total bilirubin $0.49 \mathrm{mg} / \mathrm{dL}$, aspartate aminotransferase $85 \mathrm{IU} / \mathrm{L}$, alanine aminotransferase $71 \mathrm{IU} / \mathrm{L}$, alkaline phosphatase $368 \mathrm{IU} / \mathrm{L}$, gamma glutamyl transferase $174 \mathrm{U} / \mathrm{L}$, albumin $3.84 \mathrm{~g} / \mathrm{dL}$, and normal clotting tests. Portal Doppler endoscopy study revealed changes suggestive of portal hypertension without signs of portal thrombosis. During hospitalization, the platelet count continued to decrease, requiring platelet transfusion. In order to rule out the presence of liver damage, an abdominal magnetic resonance imaging (MRI) was requested, which reported the presence of simple liver cysts and changes by metastases resection, associated with findings suggestive of portal hypertension (splenomegaly and mild ascites), in the absence of liver cirrhosis.

Diagnosis of non-cirrhotic portal hypertension was made, which was attributed to treatment with oxaliplatin. Chemotherapy was suspended and deferred until further endoscopic control, and an exhaustive genomic profiling was requested to define management of subsequent lines. The patient was discharged in the absence of new bleeding episodes and stability in the platelet count.

\section{Discussion}

Portal hypertension is defined as an elevated portal venous pressure gradient greater than $5 \mathrm{~mm} \mathrm{Hg}$ [4], with symptoms developing when pressures are above $10 \mathrm{~mm} \mathrm{Hg}$, which is called clinically significant portal hypertension. It is classified as prehepatic, hepatic and post-hepatic, with hepatic causes divided into pre-sinusoidal, sinusoidal and post-sinusoidal depending on the level of resistance in the portal venous system [3].

Sinusoidal portal hypertension in the majority of cases is associated to cirrhosis; however there are other cases presented in patients without this association, an entity known as NCPH. It is a pre-sinusoidal cause of portal hypertension in which hepatic venous pressure gradient is almost always normal but the intrasplenic and intra variceal pressures are increased with patency of the hepatic and portal veins. Clinically it is characterized by signs and symptoms of portal hypertension such as splenomegaly and preserved liver function. Most common presentation includes symptoms related to complications of portal hypertension such as variceal bleed, long-standing splenomegaly, anemia and thrombocytopenia secondary to hypersplenism, ascites, and circulatory dysfunction [4].

The pathophysiology is not well understood. Animal models and pathogenetic theories have been described suggesting immunologic, infective and vascular bases of the disease. Prothrombotic states, ADAMTS13, infections, human immunodeficiency virus (HIV), genetic, immunologic and immune deficiencies are possible causes. Among causes of $\mathrm{NCPH}$, drug toxicity must be questioned. Several drugs have been associated to it, with methotrexate and amiodarone being the most common [3]. Other causes have been described such as arsenic, 6-mercaptopurine, azathioprine, didanosine, vitamin A, irradiation and herbs.

Among chemotherapeutic agents, oxaliplatin is one of the reported drugs known to cause portal hypertension. It must be suspected in patients without previous known liver disease, who manifest with ascites, splenomegaly, esophageal varices or thrombocytopenia (in the absence of other blood cell abnormalities, and not attributed to bone marrow toxicity secondary to chemotherapy).

Despite not being clear about the pathophysiological process that causes this association, considering the low number of reports, it is believed to be secondary to the sinusoidal damage it exerts. This could be explained by oxaliplatin-induced toxicity to the endothelium of the hepatic sinusoid, by increasing fenestrations and porosity in endothelial cells [5]. Subsequently, these cells are then displaced into the lumen of the sinusoid, causing obstruction to blood flow, with a consequent increase in portal pressure, an entity known as sinusoidal obstruction syndrome. However, this theory has been explained only in murine models, with human studies still lacking [6].

Sinusoidal obstruction syndrome (formerly known as veno-occlusive liver disease) is characterized by findings suggestive of portal hypertension (ascites, edema, collateral circulation), but it is usually accompanied by jaundice, abdominal pain, and painful hepatomegaly [7]; clinical manifestations which our patient did not have. For this reason, we consider there may be some other mechanisms involved in the development of oxaliplatin-induced $\mathrm{NCPH}$ that have not yet been fully 
elucidated.

Despite not knowing all the mechanisms by which NCPH occurs, some series have demonstrated the high prevalence of splenomegaly and thrombocytopenia in patients with colorectal carcinoma treated with the FOLFOX, concluding that this complication is dose-dependent, and that additional studies are required to explain this causality $[8,9]$.

Finally, regardless of the pathophysiological mechanisms, the treatment of NCPH and its complications is based on the suspension of oxaliplatin and the primary and secondary prophylaxis according to the manifestations of each patient, with beta-blockers being the cornerstone of variceal bleeding, as in this case.

\section{Conclusions}

In patients under treatment with oxaliplatin who debut with ascites, variceal bleeding, splenomegaly or thrombocytopenia, drug-induced NCPH must be always ruled out. Despite of not being common, oxaliplatin-induced portal hypertension must be suspected in patients receiving chemotherapy. Underdiagnosis can be life-threatening, and in case of symptoms, we believe oxaliplatin should be discontinued, weighing riskbenefit for patients, and other treatment alternatives should be inquired.

\section{Acknowledgments}

None to declare.

\section{Financial Disclosure}

No funding was received.

\section{Conflict of Interest}

None to declare.

\section{Informed Consent}

The manuscript has been sufficiently de-identified to protect the patient. However, her informed written consent was obtained for the publication of the case.

\section{Author Contributions}

All authors contributed to the editing of the manuscript. MCFL wrote the manuscript. $\mathrm{EB}, \mathrm{AG}$ and $\mathrm{WM}$ contributed to the manuscript corrections.

\section{Data Availability}

The authors declare that data supporting the findings of this study are available within the article

\section{References}

1. Benson AB, Venook AP, Al-Hawary MM, Cederquist L, Chen YJ, Ciombor KK, Cohen S, et al. NCCN guidelines insights: colon cancer, version 2.2018. J Natl Compr Canc Netw. 2018;16(4):359-369.

2. International Agency For Cancer Research. World Health Organization. 2021. Cancer Today. Available at: https:// gco.iarc.fr/today/online-analysispie? $\mathrm{v}=2018 \& \mathrm{amp} ; \mathrm{m}$ ode $=$ cancer\&amp;mode_population $=$ continents\&am p;population $=900 \& a m p ;$ populations $=170 \& a m p ; k e y$ $=$ total $\& a m p ; s e x=0 \& a m p ;$ cancer $=39 \& a m p ;$ type $=1 \& a$ $\mathrm{mp} ;$ statistic $=5 \& \mathrm{amp} ;$ prevalence $=0 \& \mathrm{amp} ;$ population group $=0 \&$ amp;ages_group $\% 5 \mathrm{~B} \% 5 \mathrm{D}=0 \& \mathrm{amp} ;$ ages group $\% 5 \mathrm{~B} \% 5 \mathrm{D}=17 \& \mathrm{amp} ; \mathrm{nb}$ items $=7 \& \mathrm{amp} ;$ group $_{-}$ cancer $=1$ \&amp;include_nmsc $=1$ \&amp; include_nmsc other $=1 \&$ amp; half_pie $=0$ \&amp; donut $=0 \&$ amp; populati on_group_globocan_id=\#collapse-group-0-1. Accessed on September 1, 2020.

3. Labianca R, Nordlinger B, Beretta GD, Mosconi S, Mandala M, Cervantes A, Arnold D, et al. Early colon cancer: ESMO Clinical Practice Guidelines for diagnosis, treatment and follow-up. Ann Oncol. 2013;24(Suppl 6):vi6472 .

4. Khanna R, Sarin SK. Non-cirrhotic portal hypertension - diagnosis and management. J Hepatol. 2014;60(2):421441.

5. Bloom S, Kemp W, Lubel J. Portal hypertension: pathophysiology, diagnosis and management. Intern Med J. 2015;45(1):16-26.

6. DeLeve LD, Ito Y, Bethea NW, McCuskey MK, Wang $\mathrm{X}, \mathrm{McCuskey} \mathrm{RS.} \mathrm{Embolization} \mathrm{by} \mathrm{sinusoidal} \mathrm{lining} \mathrm{cells}$ obstructs the microcirculation in rat sinusoidal obstruction syndrome. Am J Physiol Gastrointest Liver Physiol. 2003;284(6):G1045-1052.

7. Slade JH, Alattar ML, Fogelman DR, Overman MJ, Agarwal A, Maru DM, Coulson RL, et al. Portal hypertension associated with oxaliplatin administration: clinical manifestations of hepatic sinusoidal injury. Clin Colorectal Cancer. 2009;8(4):225-230.

8. Fan CQ, Crawford JM. Sinusoidal obstruction syndrome (hepatic veno-occlusive disease). J Clin Exp Hepatol. 2014;4(4):332-346.

9. Politano S, Pathak P, Hoff PM, Charnsangavej C, Overman MJ, Loyer E, Vauthey J, et al. The use of 5-fluorouracil and oxaliplatin (FOLFOX) for colorectal cancer is associated with the development of splenomegaly and thrombocytopenia. J Clin Oncol. 2008;26(15_suppl):4102-4102. 